\title{
Developments in Neural Vision Technology
}

\author{
Alan M. Litke ${ }^{1}$ \\ Santa Cruz Institute for Particle Physics \\ University of California \\ Santa Cruz, CA, USA \\ E-mail: alan.litke@cern.ch
}

The visual system is a remarkable neural network that is able to extract vital information about the external visual world. The conversion of the dynamic visual input into processed electrical signals is performed by the retina, an extraordinary biological pixel detector that lines the back of the eye and transmits coded information to the brain. The brain does further processing and generates visual perceptions. In this report, after an introduction to the visual system, I will describe the technology and experimental methods we have developed and employ, in close collaboration with neurobiologists, to probe both the retina and the brain. These methods are based on large-scale multielectrode array systems that can record, and in some cases stimulate, the electrical activity of a large population of neurons. This project was inspired by the development of silicon microstrip detectors for high energy physics experiments.

24th International Workshop on Vertex Detectors -VERTEX2015-

1-5 June 2015

Santa Fe, New Mexico, USA

\section{${ }^{1}$ Speaker}




\section{Introduction}

In this report I will give an overview of technological developments used to study visual processing by the retina and the brain. These developments were very much inspired by silicon microstrip detector technology and the VERTEX International Workshops [1].

We would like to understand the language used by the eye to send information about the outside visual world to the brain and we would like to know what the brain does with the information it receives from the eye. In this context I will discuss the visual system and the retina, the Retina Project, the Mouse Brain Project, and the Retinal Prosthesis Project, and finally give a summary and an outlook.

The visual system is illustrated in Fig. 1a. The outside visual world is focused by the cornea and lens of the eye onto a biological pixel detector (the retina) that converts the incident photons into electrical signals and performs a first stage of image processing. The output electrical signals are transmitted to the brain through a multi-fiber cable (the optic nerve) to a relay station (the lateral geniculate nucleus) and then onto the primary visual cortex for further processing.

The retina, illustrated in Fig. 1b, is a thin neural tissue ( $\sim 300 \mu \mathrm{m}$ thick) composed of the photoreceptors (rods and cones), three processing layers (horizontal, bipolar and amacrine cells), and the output cells (the retinal ganglion cells - RGCs). The output cables (the axons) from the ganglion cells form the optic nerve.

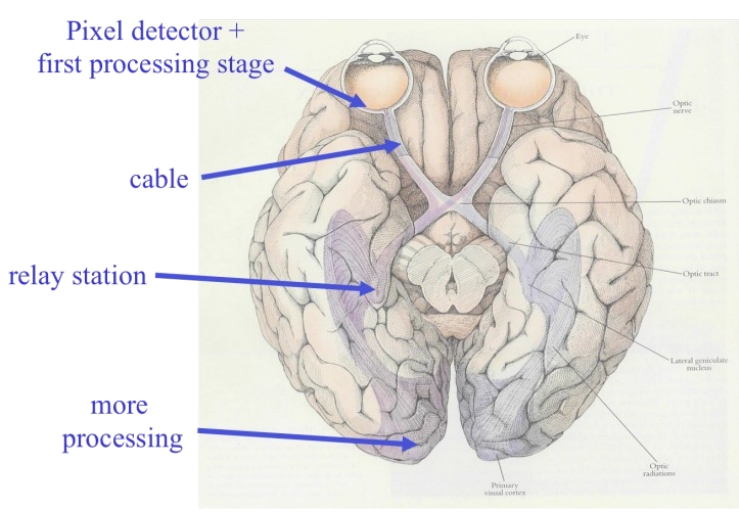

Figure 1a. The primate visual system.

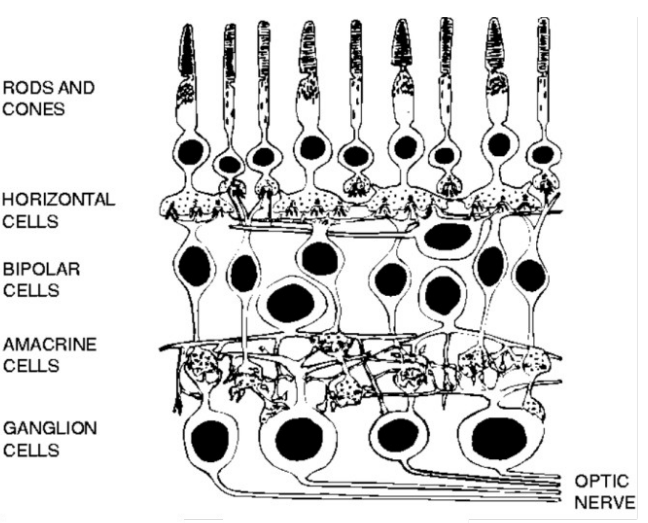

Figure 1b. A cross-section of the retina.

\section{The Retina Project}

The goal of the Retina Project is to understand how the retina processes and encodes dynamic visual images. The experimental method employed is to record the patterns of electrical activity ("spikes") generated by hundreds of retinal output neurons (the RGCs) as they respond to a movie focused on the input neurons (the photoreceptors). This is schematically illustrated in Fig. 2a. Of course, the brain does something remarkable: it extracts from the $\sim 1$ million spike trains (from each human retina) the critical information it needs about the external visual world. How the brain does this is very poorly understood. 
The technology we use is based on silicon microstrip detector techniques and expertise developed for high energy physics experiments (including the search for the Higgs Boson). This is very much an example of the application of expertise in high energy physics instrumentation to neurobiology.

The measurement of the functional properties of the retina, based on the work of Meister, Pine and Baylor [2], is illustrated in Fig. 2b. Live retinal tissue is placed, RGC layer down, in a chamber glued onto on a glass substrate. The chamber is filled with oxygenated physiological saline solution; under these conditions the tissue can be kept alive for several hours. A movie (a dynamic visual stimulus), displayed on a computer monitor, is focused on the retinal photoreceptors. The electrical signals generated by the RGCs, as the retina responds to the visual stimulus, are picked up by a two-dimensional array of microscopic electrodes fabricated on the top surface of the glass substrate.

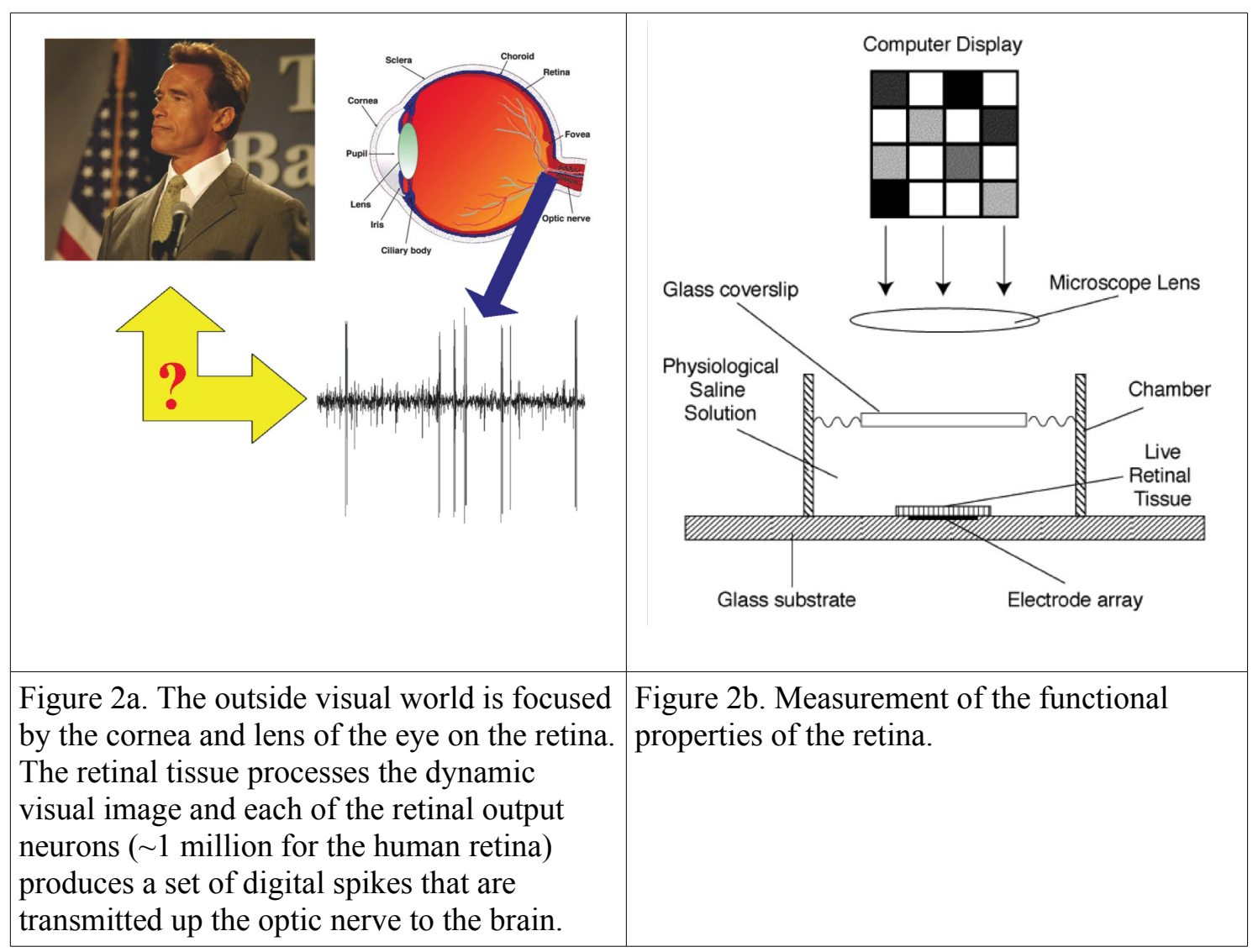

Fig. 3a shows a cross section of the multielectrode array (MEA). The electrodes and conducting traces are made of indium tin oxide (ITO); the insulating layer is silicon nitride. The electrodes are electroplated with platinum to reduce the impedance of the electrode/saline solution interface.

The MEAs come in a variety of geometries, as shown in Fig. $3 b$. The "1 electrode" array represents the traditional method of neurobiology: recording from one neuron with one electrode. The "61 electrodes" geometry represents the previous state-of-the-art [2,3], with $60 \mu \mathrm{m}$ inter-electrode spacing, and can record from tens of RGCs simultaneously. The 512 electrodes MEA is our current standard [4], also with $60 \mu \mathrm{m}$ inter-electrode spacing; it can 
simultaneously record from hundreds of RGCs. For studies of small-area RGCs, the highdensity MEA with $30 \mu \mathrm{m}$ spacing [5] can be used; for large-area retinal neurons (including spiking amacrine cells) large area MEAs with $120 \mu \mathrm{m}$ spacing have been utilized [6]. The next generation of MEA systems we hope to implement will increase the number of electrodes by a factor of four to $\sim 2048$, with 30 and $60 \mu \mathrm{m}$ spacing.

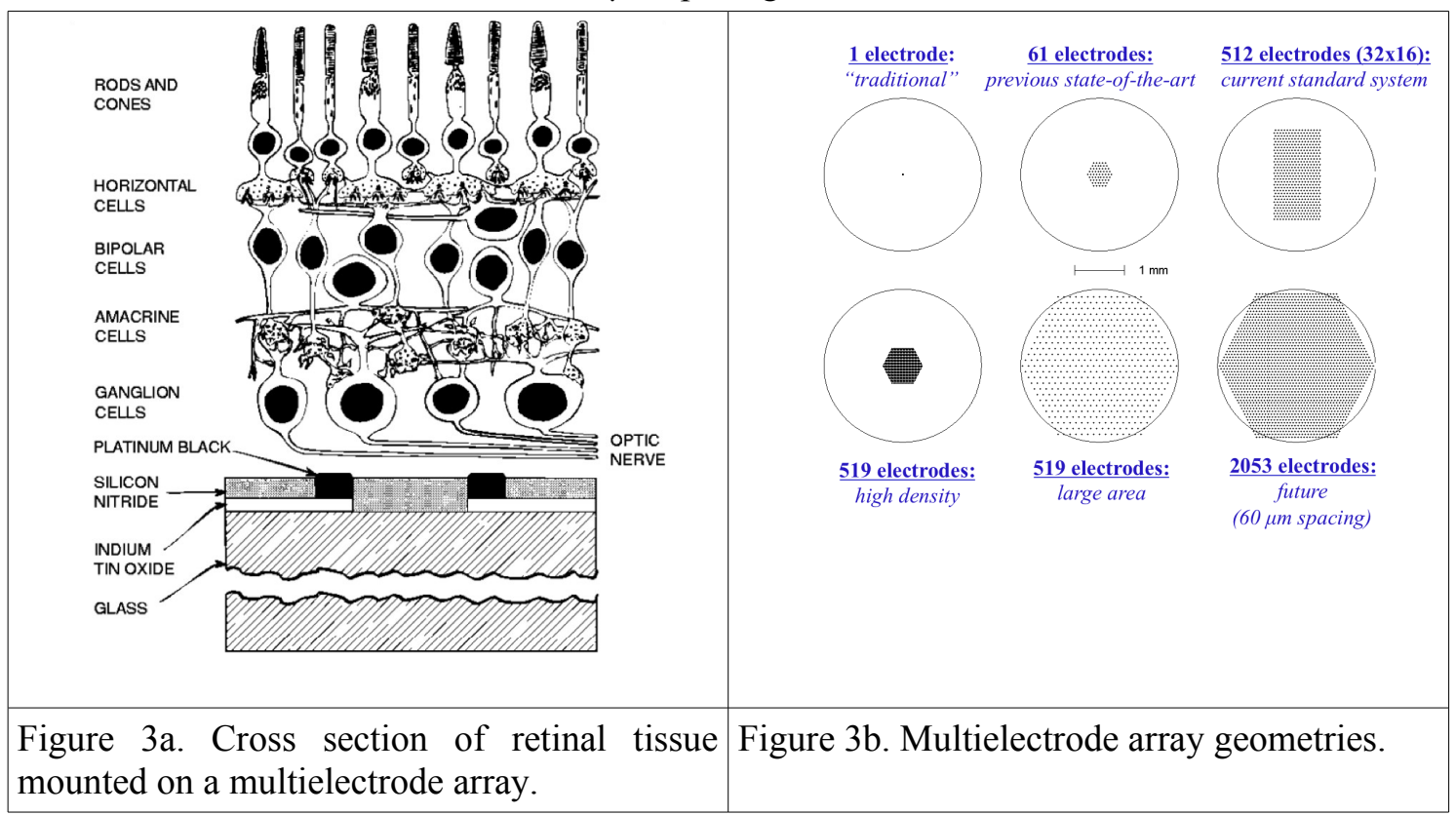

A photo of a section of a 512-electrode array is displayed in Fig. 4a. The MEAs mentioned so far have been planar arrays. For studies of acute brain slices Gunning has fabricated bed-of-needles arrays, with needles to penetrate into the interior volume of the brain slice (Fig. 4b) [7]. Each needle has a conducting tungsten/polysilicon core, surrounded by a silicon dioxide insulating layer, and an exposed, conducting electrode tip.

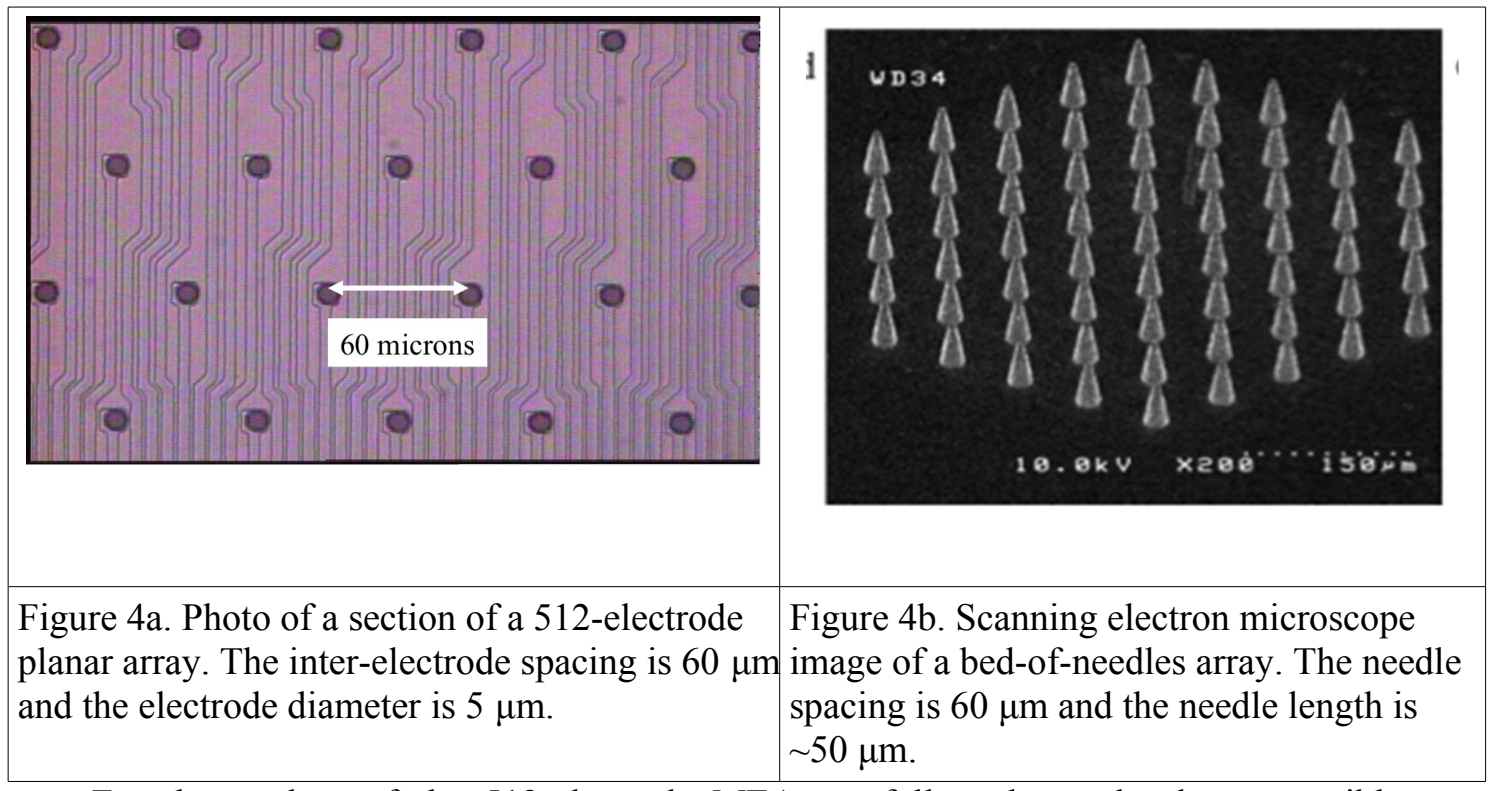

For the readout of the 512-electrode MEA we followed, as closely as possible, our development of the Silicon Strip Vertex Detector (SSVD, Fig. 5a) for the MARK II experiment 
at the SLAC Linear Collider [8]. Each microstrip detector module, after all, had 512 strips to read out. The key ingredients for the readout of the SSVD were the Microplex readout chip of Walker, Parker and Hyams [9], with 128 amplifier channels and multiplexed output, and highdensity wire-bonding (Fig. 5b).

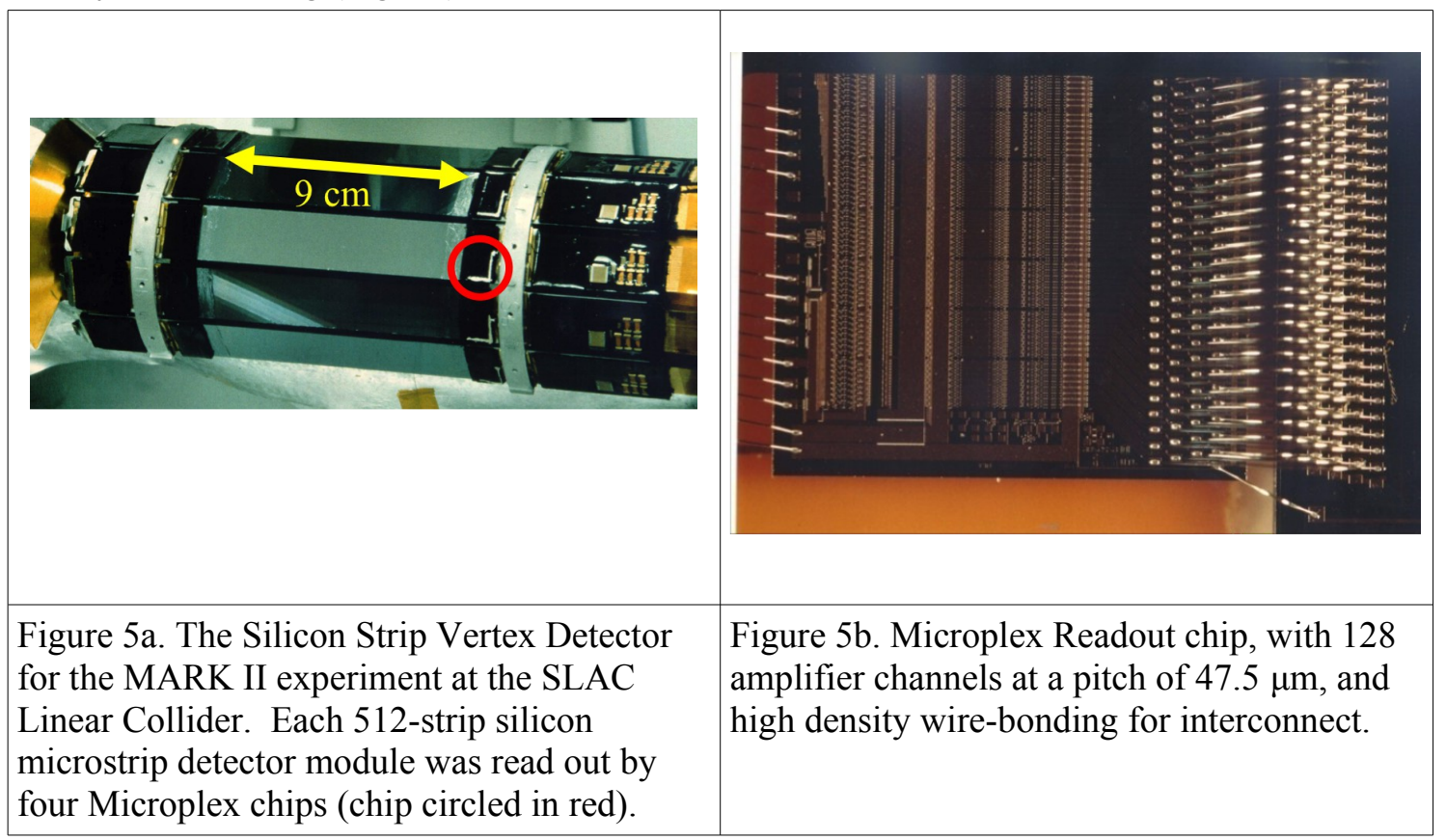

The "Neuroboard" [4] is shown in Fig. 6a. The retinal tissue is placed in the chamber that is glued on top of the 512-electrode MEA. A platinum wire that serves as the reference electrode is placed around the inner circumference of the chamber. The MEA is wire-bonded to fan-ins and eight pairs of 64-channel chips for readout. The Platchip [10] provides AC coupling and current sources for electroplating and neuron electrical stimulation. The Neurochip [10] provides amplification, filtering and multiplexing. These chips were designed by the Dabrowski group in Krakow.

We have used the Neuroboard for a wide variety of retinal studies in collaboration with neurobiologists, particularly the Chichilnisky group at the Salk Institute (now at Stanford). One result is illustrated in Fig. 6b. With the ability to record from hundreds of RGCs in a single preparation, with fine spatial and temporal resolution, we can identify and characterize rare functional cell classes that have not been previously studied. The example illustrated in Fig. 6b is the "upsilon" cell that we characterized, for the first time, in the primate retina [11]. It has functional properties that appear to be well-suited for the detection of moving objects or moving textured patterns.

\section{The Mouse Brain Project}

The goal of the Mouse Brain Project is to understand how the mouse brain processes and encodes dynamic visual images. We choose the mouse because of the remarkable set of genetic and molecular biology tools available for the study of the mouse brain. The method we employ, following the work of Niell and Stryker [12], is to record the patterns of brain activity 
of an awake, behaving mouse as the mouse watches a movie. The recording technology we use is based on thin, multi-shank multielectrode silicon probes.

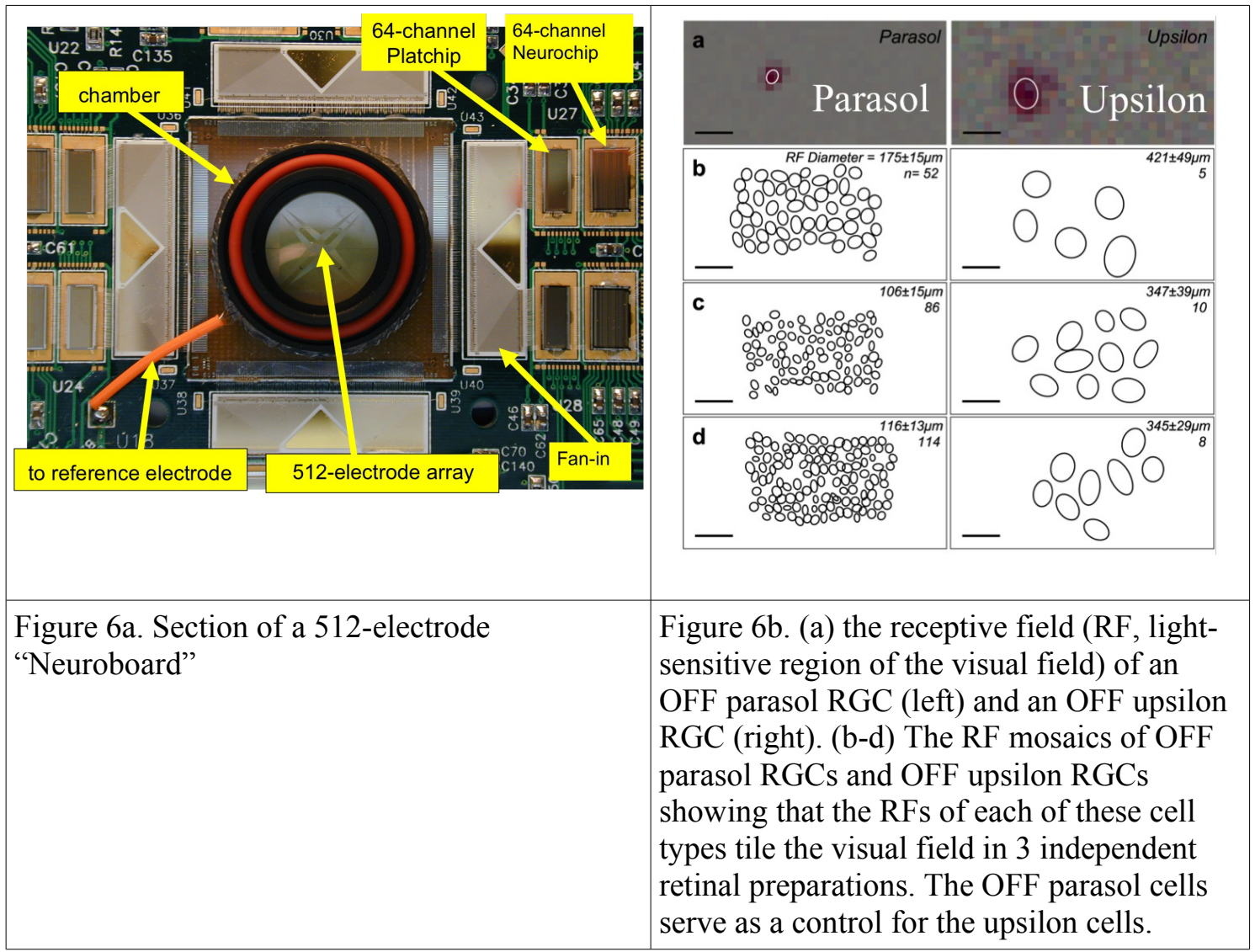

The potential applications of this method include: (1) measurement of the functional properties of visually responsive neurons; (2) study of neural system properties such as connectivity, circuitry and rhythms; (3) study of visual perception and behavior; (4) study of these cellular and system properties with transgenic mice; and (5) biomedical applications, with transgenic mouse models of neurological disorders.

A 64-electrode silicon probe [13], kindly supplied by Masmanidis (UCLA), is shown in Figs. 7a,b. The probe was wire-bonded and assembled into a recording module (Fig. 7c) by the Santa Cruz Institute for Particle Physics (SCIPP) technical staff (Martinez-McKinney and Kachiguin).

\begin{tabular}{|l|l|l|}
\hline & \\
\hline
\end{tabular}


Fig. 8 illustrates the placement of two and four shank probes with 128 or 256 electrodes in two visually responsive mouse brain regions - the superior colliculus (8a) and the primary visual cortex (8b). (These probes will be kindly supplied by Masmanidis.)

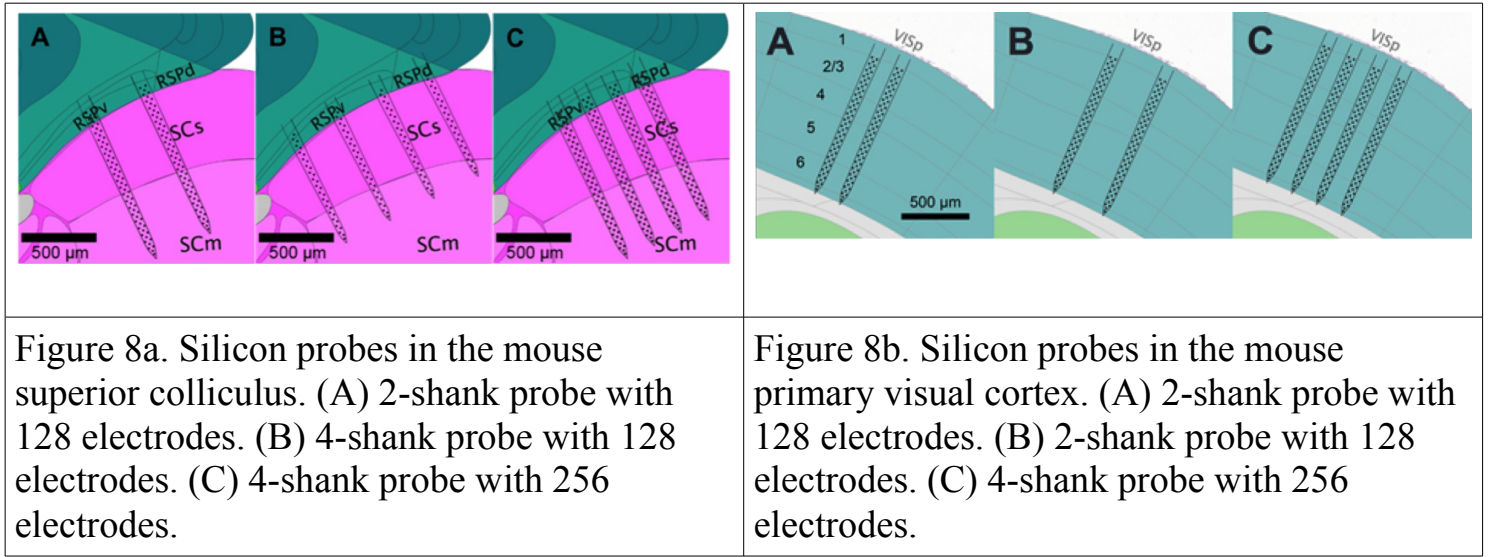

Fig. 9a shows an assembled silicon probe recording module with a probe board (onto which the silicon probe is glued and wire-bonded); an interface board; and a 64-channel headstage, with amplification, filtering, multiplexing and an ADC (available commercially from Intan Technologies). The headstage output provides the 16-bit digitized signals from each of the 64 input channels at a sampling rate up to $30 \mathrm{kHz}$ per channel.

Fig. 9b illustrates the mouse on a spherical treadmill (a freely-floating Styrofoam ball suspended by airflow [14]) as the mouse watches a movie displayed on a computer monitor. The head of the mouse is fixed and the silicon probe is inserted into the brain to record the brain activity [12]. The in vivo brain recording facility at UCSC was assembled by Ito [15].

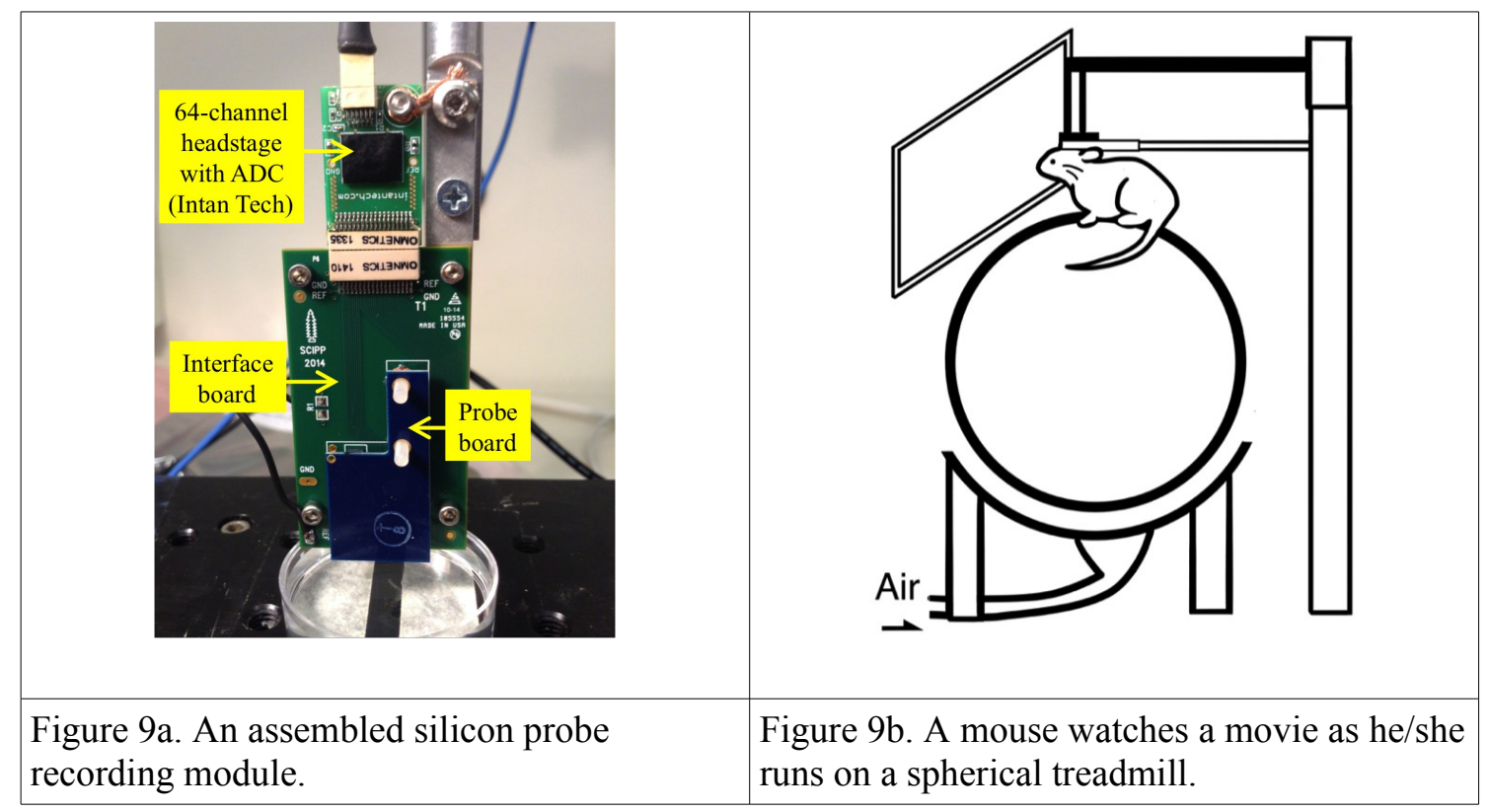

Fig. 10 shows some first results coming from this new facility, with data taken and analyzed by Ito [15]. The mouse watches a contrast-modulated movie with defined spatiotemporal properties (Fig. 10, bottom) [16]. The contrast is sinusoidally modulated with a 
10 second period. In a 10 second interval the movie goes from a gray frame (zero contrast) to a frame with high contrast and back to a gray frame. In a recording from the mouse superior colliculus with a 4-shank/128-electrode probe, the firing rate of most neurons was stimulated by contrast (top histogram) but a few neurons $(\sim 6 \%)$ had a firing rate that was suppressed by contrast (bottom histogram). These neurons like to fire when there is little going on visually. Suppressed-by-contrast neurons have been observed in the mouse retina and in other mouse brain regions, but this is the first observation of such cells in the mouse superior colliculus.

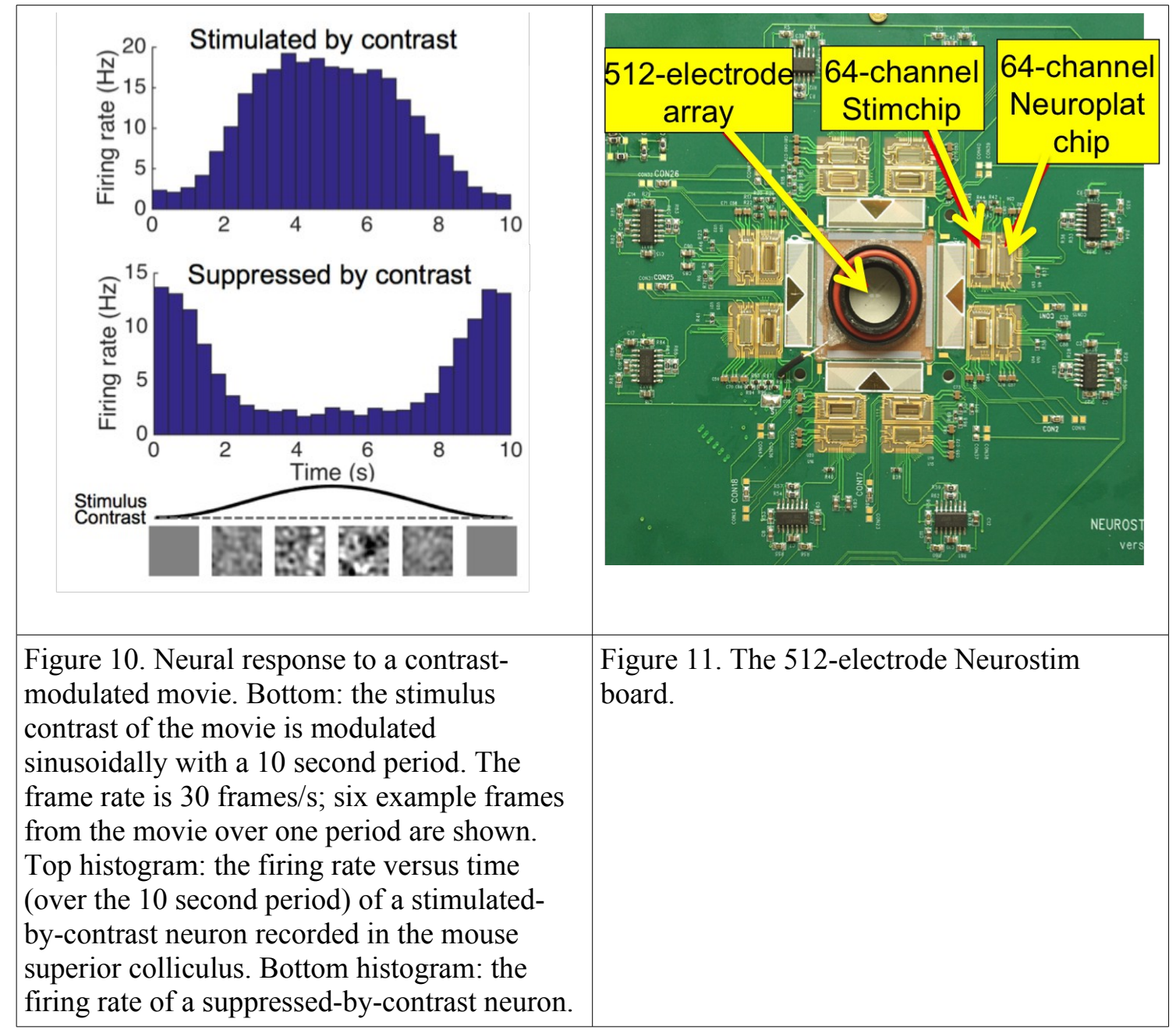

\section{The Retinal Prosthesis Project}

The goal of the Retinal Prosthesis Project has been to undertake electrophysiological studies (with isolated retina) to guide the design of retinal prosthetic devices. These devices are employed to provide some visual functionality to individuals with diseases that cause blindness due to photoreceptor degeneration. Examples of such diseases are retinitis pigmentosa and agerelated macular degeneration.

We note that a retinal prosthetic device (Argus II) was approved in 2013 by the US Federal Drug Administration as a humanitarian device for the treatment of retinitis pigmentosa [17]. This device is comprised of: (1) a video camera, mounted on glasses worn by a blind individual, 
that converts the visual image into a digital image; (2) a processor that converts the digital image into a pattern of electrical stimulation pulses; (3) a RF transmitter/receiver that transmits the stimulation data through the skull; and (4) an implanted electrode array that applies the electrical stimulation pulses to the RGCs. These electrical pulses can then stimulate the nearby RGCs to generate a pattern of spikes that are sent through the optic nerve to the brain. The goal is to replicate, by this electrical stimulation, the spiking activity pattern that a healthy retina would generate when subject to the same visual scene. The Argus II array, however, has a $6 \mathrm{x}$ 10 set of electrodes with a $200 \mu \mathrm{m}$ electrode diameter and an electrode spacing of 500-650 $\mu \mathrm{m}$. These dimensions are large compared to the scale necessary to stimulate individual RGCs $(\sim 10$ $\mu \mathrm{m})$. The net result is limited visual functionality for the individual.

What then can be achieved with a high-density array of small diameter $(\sim 5-10 \mu \mathrm{m})$ electrodes, such as we have used for our retinal studies? To evaluate this issue we have developed 61 and 512 electrode MEA systems $[18,19]$ that can stimulate RGC activity with a very general spatiotemporal pattern of electrical stimulation pulses to replicate, as faithfully as possible, the RGC activity pattern expected for the input visual image. These systems can also simultaneously record the generated spike waveforms on each electrode channel. Thus we can evaluate how the neural system responds to the patterned stimulation.

The stimulation/recording systems use 61 and 512-electrode MEAs (with 30 or $60 \mu \mathrm{m}$ inter-electrode spacing) combined with a set of 64-channel "Stimchips" [20] and "Neuroplat" chips [21] developed by the Dabrowski group at the AGH University of Science and Technology in Krakow. The Stimchip, designed and applied to retinal prosthesis studies by Hottowy, includes 64 independent digital-to-analog converters for current or voltage stimulation, and circuitry for suppression of the electrical artifact. A photo of a 512-electrode "Neurostim" board is shown in Fig. 11.

In studies with a 61-electrode Neurostim system applied to a rat retina we were able to replicate a visually-evoked spiking pattern for multiple neurons with a spatio-temporal pattern of electrical stimulation pulses applied by multiple electrodes. The replication was achieved with high stimulation efficiency ( $\sim 99 \%)$ of individual RGCs and excellent time resolution ( $\sim 50$ $\mu$ ) [18]. Similar results have been achieved with a 61-electrode Neurostim system applied to primate retina [22]. However, three issues (among others) remain to be resolved. First, stimulation of an RGC of one cell type may stimulate one or more nearby RGCs of the same cell type. With small-diameter electrodes this is not a major problem as the stimulated RGCs will respond in the same way and be well-localized. Second, stimulation of an RGC of one cell type may stimulate one or more RGCs of different cell types. For faithful replication of the RGC spiking pattern we should stimulate RGCs of each cell type independently, according to how that specific cell type would respond to the input visual stimulus. Work on cell-type selective activation has been carried out with primate retina using single electrode stimulation [23] and two or three neighboring electrodes for stimulation [24]. Third, stimulation of an RGC may also stimulate a nearby axon from a distant cell or a bundle of axons from multiple distant cells. These axonal signals will be interpreted by the brain as originating from the distant RGC(s), giving non-local perceptual effects. Studies are on-going, with a 512-electrode Neurostim system, to develop stimulation methods to activate RGCs, in primate retina, without simultaneously activating axons or axonal bundles [25]. 


\section{Summary and Outlook}

I have taken you on a journey that started with high energy physics and the development of silicon microstrip detectors, to the study of image processing by the retina, then onto image processing by the brain, and lastly to biomedical applications including retinal prosthesis studies and, we hope, future studies on neurological disorders. As genetics, molecular biology, optical and electrophysiological methods to stimulate and record neural system activity, brain imaging, computational neuroscience and "big data" methods are all advancing at a rapid pace and converging on the study of the brain, we are at the beginning of a neuroscience revolution. President Obama's BRAIN Initiative (Brain Research through Advancing Innovative Neurotechnologies), roughly modeled on the Human Genome Initiative, and the Human Brain Project (funded by the European Union) give just a hint of the ongoing and future activities. This is an exciting time for collaborations of physicists, biologists, engineers, mathematicians and computer scientists to help understand how neural systems develop, how they process and encode information, and what can be done when they malfunction.

\section{Acknowledgements}

I would like to gratefully acknowledge the many contributions of my many collaborators in physics, multielectrode array and silicon probe fabrication, integrated circuit design and neurobiology:

Santa Cruz Institute for Particle Physics (SCIPP)/UC Santa Cruz (electrode array fabrication, Neuroboard design and implementation, data acquisition and analysis software, and the in vivo Brain Recording Facility): A. Grillo, M. Grivich, S. Ito, S. Kachiguine, F. Martinez-McKinney, D. Petrusca, A. Sher.

AGH U. of Science and Technology, Krakow (IC design and retinal prosthesis): W. Dabrowski, P. Hottowy.

$\underline{U}$. Glasgow $\rightarrow U$. Strathclyde (electrode array and bed-of-nails fabrication): D. Gunning, K. Mathieson.

$\underline{\boldsymbol{U C L A} A}$ (silicon probe fabrication): S. Masmanidis.

$\underline{S L A C}$ (bed-of-needles prototype fabrication): C. Kenney.

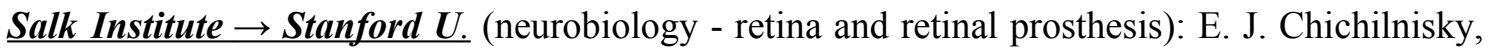
G. Field, J. Gauthier, M. Greschner, L. Grosberg, L. Jepson, P. Li, J. Shlens.

MCD Biology/UC Santa Cruz (neurobiology - in vivo mouse brain): B. Chen, D. Feldheim, Y. Zuo.

Indiana $U_{\text {. }}$ (neurobiology - in vitro brain slices): J. Beggs.

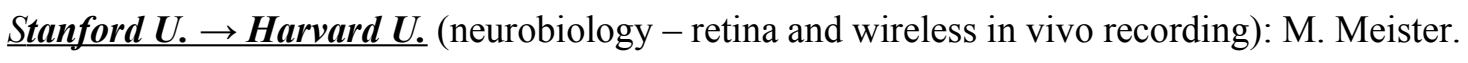

$\underline{\boldsymbol{U C S F}}$ (neurobiology - in vivo mouse brain): M. Stryker.

I would also like to gratefully acknowledge my funding sources: National Science Foundation, National Institutes of Health, McKnight Foundation, and UC Santa Cruz. 


\section{References}

[1] A. M. Litke, The retinal readout system: an application of microstrip detector technology to neurobiology, Nucl. Instr. and Meth. In Phys. Res. A 418 (1998) 203-209.

[2] M. Meister, J. Pine, D. A. Baylor, Multi-neuronal signals from the retina: acquisition and analysis, Journal of Neuroscience Methods 51 (1994) 95-106.

[3] A. Litke, M. Meister, The Retinal Readout Array, Nucl. Instr. and Meth. In Phys. Res. A 310 (1991) 389-394.

[4] A. M. Litke et al., What Does the Eye Tell the Brain?: Development of a System for the Large-Scale Recording of Retinal Output Activity, IEEE Transactions on Nuclear Science 51 (2004) 1434-1440.

[5] D. E. Gunning et al., Performance of ultra-high-density microelectrode arrays, Nucl. Instr. and Meth. In Phys. Res. A 576 (2007) 215-219.

[6] M. Greschner et al., A polyaxonal amacrine cell population in the primate retina, The Journal of Neuroscience 34, (2014) 3597-3606.

[7] D. E. Gunning et al., Dense arrays of micro-needles for recording and electrical stimulation of neural activity in acute brain slices, J. Neural Eng. 10 (2013) 016007.

[8] C. Adolphsen et al., The MARK II silicon strip vertex detector, Nucl. Instr. and Meth. In Phys. Res. A 313 (1992) 63-102.

[9] J. T. Walker et al., Development of High Density Readout for Silicon Strip Detectors, Nucl. Instr. and Meth. In Phys. Res. A 226 (1984) 200-203.

[10] W. Dabrowski et al., Development of front-end ASICs for imaging neuronal activity in live tissue, Nucl. Instr. and Meth. In Phys. Res. A 541 (2005) 405-411.

[11] D. Petrusca et al., Identification and characterization of a Y-like primate retinal ganglion cell type, J. Neurosci. 27 (2007) 11019-11027.

[12] C. M. Niell, M. P. Stryker, Modulation of visual responses by behavioral state in mouse visual cortex, Neuron 65 (2 01010$)$ 472-479.

[13] J. Du et al., Multiplexed, High Density Electrophysiology with Nanofabricated Neural Probes, PLoS ONE $6\left(\begin{array}{llll}2 & 0 & 1 & 1\end{array}\right)$ e26204.

[14] D. A. Dombeck et al., Imaging Large-Scale Neural Activity with Cellular Resolution in Awake, Mobile Mice, Neuron 56 (2007) 43-57.

[15] S. Ito, D. A. Feldheim, A. M. Litke, Large-scale silicon probe recordings identify new cell types and gamma rhythm properties in the mouse superior colliculus. Program No. 414.11, 2015 Neuroscience Meeting Planner. Washington, DC: Society for Neuroscience, 2015. Online.

[16] C. M. Niell, M. P. Stryker, Highly selective receptive fields in mouse visual cortex, J. Neurosci. 28 (2008) 7520-36.

[17] L. da Cruz et al., The Argus II epiretinal prosthesis system allows letter and word reading and longterm function in patients with profound vision loss, British Journal of Ophthalmol. 97 (2013) 632636.

[18] P. Hottowy et al., Properties and application of a multichannel integrated circuit for low-artifact, patterned electrical stimulation of neural tissue, J. Neural Eng. 9 (2012) 066005. 
[19] P. Hottowy et al., 512-electrode MEA System For Spatio-Temporal Distributed Stimulation and Recording of Neural Activity, Proc. 7th Int. Meeting on Substrate-Integrated Micro Electrode Arrays (2010, Reutlingen, Germany) 327-330.

[20] P. Hottowy et al., An integrated multichannel waveform generator for large-scale spatio-temporal stimulation of neural tissue, Analog Integr. Circuits Signal Process. 55 (2008) 239-48.

[21] P. Grybos et al., Neuroplat64-low noise CMOS integrated circuit for neural recording applications, Proc. 5th Int. Meeting on Substrate-Integrated Micro Electrode Arrays (2006, Reutlingen, Germany) 208-9.

[22] L. H. Jepson et al., High-fidelity reproduction of spatiotemporal visual signals for retinal prosthesis, Neuron 83 (2014) 87-92.

[23] L. H. Jepson et al., Focal electrical stimulation of major ganglion cell types in the primate retina for the design of visual prostheses, J. Neurosci. 33 (2013) 7194-7205.

[24] L. H. Jepson et al., Spatially patterned electrical stimulation to enhance resolution of retinal prostheses, J. Neurosci. 34 (2014) 4871-81.

[25] L. E. Grosberg et al., Axon activation with focal epiretinal stimulation in primate retina, Invest. Ophthalmol. Vis. Sci. 56 (2015) 780. 